Cine
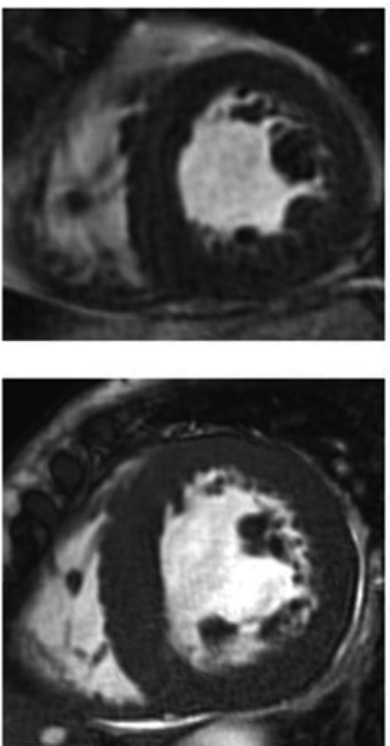

T1 Map
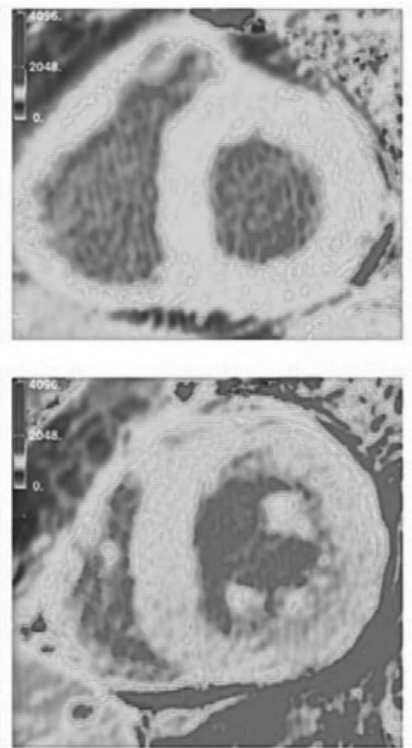

LGE
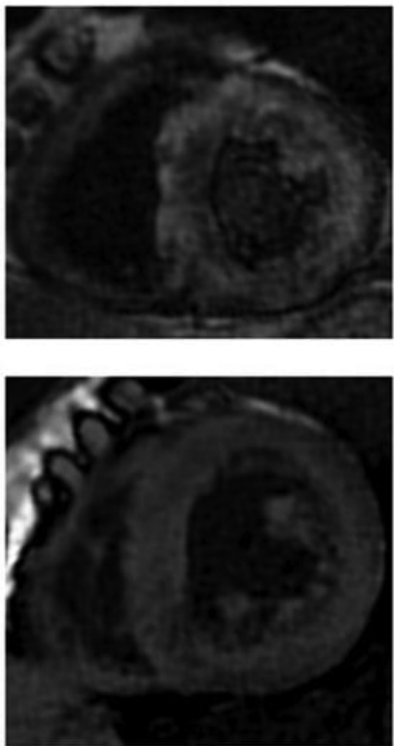

Abstract 015 Figure 2 Characteristics Examples from CMR Scans. CMR end-diastolic cine (far left), shortened modified look locker inversion recovery native $\mathrm{T1}$ map (middle) and late gadolinium enhancement (LGE) images (far right), in two patients with definite transthyretin amyloidosis (ATTR amyloidosis), one (top) with normal T1 map, mildly eleveted native myocardial T1 (1039ms) and very elevated ECV (0.7), the other (bottom) with abnormal T1 map, markedly elevated native myocardial T1 (1224ms) and very elevated ECV (0.7).

for the significance of the difference between areas under the ROC curves). During follow-up, 63 deaths occurred: 34 ATTRwt, 29 ATTRm. Whilst native T1 was not predictive of death, ECV was (HR, 1.130; 95\% confidence interval, 1.06$1.2 ; \mathrm{p}<0.001)$ and remained independent after adjustment for age, N-terminal pro b-type natriuretic peptide, left ventricular (LV) ejection fraction, E/E', LV mass index, global longitudinal strain and tricuspid annular plane systolic excursion.

Conclusions CMR-determined native myocardial T1 and ECV provide excellent diagnostic accuracy for identification of ATTR cardiac amyloidosis and both variables track DPD-determined amyloid burden well. In this study, whilst T1 was not a predictor of mortality, ECV was independently associated with mortality.

\section{USPIO-ENHANCED CMR COMPREHENSIVE METHODOLOGICAL INVESTIGATION AND APPLICATION IN ACUTE MI}

1,2Jakub Lagan, ${ }^{1}$ David Clark, ${ }^{1}$ Anna Reid, ${ }^{1}$ Gavin Lewis, ${ }^{2}$ Josephine Naish, ${ }^{2}$ James Fildes, ${ }^{2}$ Andrew Trafford, ${ }^{2}$ William Critchley, ${ }^{3}$ Erik B Schelbert, ${ }^{1}$ Matthias Schmitt, 'Phillip Foden, ${ }^{1,2}$ Christopher A Miller. 'University Hospital of South Manchester, UK; ${ }^{2}$ University of Manchester, UK; ${ }^{3}$ University of Pittsburgh, UK

\subsection{6/heartjnl-2017-311399.16}

Introduction Quantification of active myocardial inflammation may improve diagnosis, guide management and provide trial end-points for novel therapies. Ultrasmall particles of iron oxide (USPIO) are phagocytosed by activated leukocytes and USPIO-enhanced CMR is increasingly used to assess tissue inflammation. We aimed to; 1 . Compare T2* imaging with T1 mapping, which is proposed as an alternative for 'native' cardiac iron measurement; 2. Determine whether imaging at a single time point post-USPIO is sufficient to detect active accumulation in tissue; 3. Determine whether USPIO signal from infarct and remote zones in acute myocardial infarction (MI) reflects active myocardial accumulation or passive 'washthrough' in oedematous myocardium.

Methods Four healthy volunteers and six patients with acute MI underwent 1.5T CMR, including T1 and T2* mapping, before and at multiple time points following $4 \mathrm{mg} / \mathrm{kg}$ ferumoxytol.

Results Normalised T2* of spleen, an organ with high active leukocyte activity, dropped post-USPIO and remained low over the study period (Figure 1), with no correlation seen between spleen $\mathrm{T} 2 *$ and blood T1 (rho $=-0.43, \mathrm{p}=0.875)$. In comparison, T1 recovery in spleen correlated strongly with $\mathrm{T} 1$ recovery in blood (rho=0.924, $\mathrm{p}<0.001)$. In healthy myocardium, an organ with low leukocyte activity, T1 and T2*recovery both correlated strongly with blood T1 (rho=0.953, p $<0.001$; rho $=0.935, \mathrm{p}<0.001$ respectively).

In MI, absolute T2* values dropped and remained significantly lower in infarcted (15 vs $27 \mathrm{~ms}, \mathrm{p}<0.001,22$ vs $38 \mathrm{~ms}$, $\mathrm{p}=0.001)$ and remote myocardium (21 vs $27 \mathrm{~ms}, \mathrm{p}=0.05,28$ vs $38 \mathrm{~ms}, \mathrm{p}=0.024)$ compared to healthy controls. T2* and T1 recovery curves post-USPIO were significantly different in both infarcted $(p=0.028)$ and remote myocardium $(p=0.004$; Figures 2).

Conclusions $0 \mathrm{~T} 2 *$ is sensitive to active tissue accumulation of USPIO, likely because T2* reflects field gradients, such as those generated by compartmentalised (phagocytosed) USPIO. T1, which is due to short range dipolar interactions that reduce as USPIOs wash-out, simply tracks passive wash-through. T1 is therefore less suitable for detecting active leukocytes. Measuring T2* at a single time point post-USPIO is insufficient to determine tissue accumulation from passive wash-through. USPIO signal from infarct and remote zones in acute MI appears to genuinely reflect active myocardial accumulation, presumably due to phagocytosis by activated leukocytes. 